\title{
A produção científica e grupos de pesquisa sobre vigilância sanitária no CNPq
}

\author{
The scientific production and research groups \\ on sanitary surveillance at $\mathrm{CNPq}$
}

Vera Lúcia Edais Pepe ${ }^{1}$

Ana Beatriz M arinho de Noronha ${ }^{2}$

TatianaAragão Figueiredo ${ }^{1}$

Adriana deAlvarenga Linhares de Souza ${ }^{3}$

Catia Veronica dos Santos Oliveira ${ }^{1}$

Durval $M$ artins Pontes Júnior ${ }^{4}$

\footnotetext{
${ }^{1}$ Departamento de

Administração e

Planejamento em Saúde

Escola N acional deSaúde

Pública Sergio Arouca,

Fundação Oswaldo Cruz.

Rua Leopoldo Bulhões 1.480,

$7^{\circ}$ andar, M anguinhos.

21041-210 Rio deJaneiro

RJ. verapepe@ensp.fiocruz.br

${ }^{2}$ Escola Politécnica de Saúde

Joaquim Venâncio, Fundação

Oswaldo Cruz.

${ }^{3}$ Instituto de Tecnologia em

Imunobiológicos, Fundação

Oswaldo Cruz.

${ }^{4}$ Secretaria deEstado de

SaúdedeM inas Gerais.
}

Abstract Sanitary surveillance is an intersectorial and multidisciplinary practice of health regulation. The aim was to describe the scientific research on sanitary surveillance and its research groups in Brazil during the period of 1997 to 2003, through the Census of 2000, 2002 and 2004 of Directory of Research Groups of the Scientific and Technological Development National Council (CN Pq). The term "sanitary surveillance" was used to search the production and the research groupsin the LattesPlatform of CN Pq. Therewere 1,194 items, 913 in bibliographic production and 281 in post-graduated production, with an incre ment of $540 \%$ on the period. There were $735 \mathrm{re}$ search groups, created mostly from 2000 to 2003 and 6,263 researchers concentrated in the Southeast Region and in CN Pq sub area of Public H ealth. The great increase of the production lead to the conclusion that sanitary surveillance have been a locus of production only in the last decade, presented in scientific events of Public Health and until now concentrated just like others areas in $\mathrm{H}$ ealth.

Key words Sanitary surveillance, Scientific production, Research groups, Research lines, Lattes Platform
Resumo A vigilância sanitária caracteriza-sepor ser prática intersetorial e multidisciplinar, utilizando-se, de longa data, do conhecimento científico produzido em diferentes áreas. Esta pesquisa objetivou descrever a produção científica sobre vigilância sanitária registrada por pesquisadores cadastrados na Plataforma Lattes do CN Pq eanalisar o perfil dos grupos de pesquisa que trabaIham com o tema. Utilizaram-seos Censos de2000, 2002 e 2004 do Diretório dos Grupos de Pesquisa no Brasil do Conselho Nacional de Desenvolvimento Científico e Tecnológico com o termo "vigilância sanitária". A produção científica foi analisada no período de 1997 a 2003. Encontraramse 1.194 registros, sendo 913 na categoria de produção bibliográfica e 281 em orientações concluídas, com aumento de $540 \%$ no período. A produção foi realizada por 735 grupos de pesquisa, criados principalmente entre 2000 e 2003, e 6.263 pesquisadores, concentrados na região Sudeste e na pequena área da Saúde Coletiva. Conclui-se que, pelo crescimento da produção especialmente na última década, a vigilância sanitária tem se tornado locus de produção de saber e ocupado fóruns importantes de produção científica voltados à Saúde Coletiva no Brasil, sendo ela ainda concentrada, assim como outras áreas na Saúde. Palavras-chave Vigilância sanitária, Produção científica, Grupos de pesquisa, Linhas de pesquisa, Plataforma Lattes 
Introdução

A vigilância sanitária vem se constituindo como um importante campo da Saúde Coletiva. Lucchesi ${ }^{1}$ pontua que, função precípua do Estado, sua ação volta-se para a adequação do sistema produtivo de bens e serviços de interesse sanitário, e os ambientes, às demandas sociais de saúde, para os indivíduos e para a col etividade, e às necessidades do sistema de saúde. No cumprimento de seu papel de proteger a saúde dos cidadãos, a vigilância sanitária caracteriza-se por ser uma prática intersetorial, interinstitucional emultidisciplinar, utilizando-se do conhecimento científico produzido em diferentes áreas de conhecimento, dentre as quais: a farmacologia, a física, a química, o direito, a economia e a administração?2.

A estruturação da vigilância sanitária, seja como arranjo institucional de um sistema nacional, seja como objeto de análise e/ou locus produtor deconhecimento, tem sido construída mais recentemente, especialmente a partir do final da década de 1980. Embora seja uma prática antiga deSaúde Pública ${ }^{1,3}$ eembora al guns deseus objetos de interesse específicos - medicamentos, alimentos, produtos, serviços de saúdee ambiente, incluindo-se aqui o ambiente de trabalho - sejam estudados sob vários aspectos de longa data, apenas mais recentemente a vigilância sanitária tem ocupado os fóruns importantes de produção científica voltados à Saúde Coletiva.

$\mathrm{Na}$ última década, a vigilância sanitária tem feito um esforço de autorreflexão em eventos específicos da área, promovidos pela Associação Brasileira de Pós-Graduação em Saúde Coletiva (Abrasco). Foram realizados, até 2007, três simpósios brasileiros de vigilância sanitária e duas jornadas N orte-N ordeste de vigilância sanitária. Além destes, houve alguns eventos específicos promovidos pelos serviços de vigilância sanitária, como o I Fórum Estadual Intersetorial em Vigilância Sanitária, realizado em Florianópolis (SC), em 2004.

Pode-se supor que a produção de conhecimento nesse campo vem acompanhando, ainda que de forma tardia, o avanço da pesquisa científica no Brasil, que muito deve à consolidação da política de pós-graduação implantada no país na década de $1960^{4}$. 0 primeiro programa de pósgraduação stricto sensu especificamente voltado para a vigilância sanitária foi constituído mais recentemente, no Instituto $\mathrm{N}$ acional de Controle de Qualidade em Saúde, da Fundação Oswaldo Cruz (INCQS/Fiocruz), e data de 2001.

Uma importante fonte de informação sobre a produção científica no Brasil é a Plataforma
Lattes, que representa a experiência do Centro Nacional de Desenvolvimento Científico e Tecnológico (CNPq) na integração de bases de dados decurrículos ede instituições daárea de ciência e tecnologia em um único sistema de informações (http://lattes.cnpq.br/). A disponibilidade dessa fonte de informações sobre a pesquisa nacional abre oportunidades para a geração de conhecimento em Ciência \& Tecnologia 5 .

Este trabalho tem como objetivo descrever a produção científica sobre vigilância sanitária cadastrada na Plataforma Lattes do CN Pq e analisar o perfil dos grupos de pesquisa que trabaIham com o tema. Acredita-se poder com isso contribuir para a divulgação de sua produção na Saúde Coletiva. A ampliação do conhecimento sobre o próprio campo da vigilância sanitária e de sua produção científica contribuirá para a consolidação do Sistema N acional de Vigilância Sanitária, ajudando a direcionar, juntamentecom outros instrumentos, como o Censo $\mathrm{N}$ acional de Trabal hadores da Vigilância Sanitária e o Plano Diretor de Vigilância Sanitária $a^{6,7}$, os investimentos necessários à maior qualificação da força de trabal ho e do próprio processo de trabalho em vigilância sanitária.

\section{Métodos}

Utilizaram-se como fonte de dados os Censos 2000, 2002 e 2004 do Diretório de Pesquisa da Plataforma Lattes do CN Pq:

Censo 2000 - Período de 1997 a 2000.

Censo 2002 - Período de 1998 a 2001.

Censo 2004 - Período de 2000 a 2003.

O Diretório dos Grupos de Pesquisa no Brasil é um projeto desenvolvido no $\mathrm{CN} \mathrm{Pq}$ desde 1992 e registra informações sobre os grupos de pesquisa em atividade no país, com três principais finalidades, segundo a própria instituição: servir, no dia a dia do exercício profissional, de instrumento para o intercâmbio e a troca de informações entre membros da comunidade científica e tecnológica; prover informações de apoio ao planejamento eà gestão das atividades deCiência \& Tecnologia; e preservar a memória da atividadecientífico-tecnológica no Brasil. Eletambém reúneinformações sobre pesquisadores, estudantes, técnicos, linhas de pesquisa em andamento, produção científica, tecnológica e artística geradas pelos grupos.

A base de dados do Diretório dos Grupos de Pesquisa é formada por dois módulos: Base Correntee BaseCensitária. A BaseCorrente, considerada a base de dados operacional do sistema, é 
uma base de dados relacional, na qual estão armazenadas as informações dos grupos de pesquisa, cadastradas pelos seus líderes e por todos os pesquisadores por meio do Currículo Lattes. A Base Censitária, modelada para consultas gerenciais, utiliza totalizadores eaglomeraçõesoriginados da extração, análise, avaliação e consolidação de informações oriundas da base corrente e possui indexação textual, o que permite arealização deconsultas sobre dados não estruturados4.

As Bases Censitárias do Diretório dos Grupos de Pesquisa são extraídas periodicamente da base corrente (normalmente a cada dois anos). Elas representam "fotografias" da pesquisa no Brasil, tiradas em datas, previamente anunciadas pelo CN Pq para que os participantes dos grupos atualizem seus dados na Plataforma Lattes. As principais consultas disponíveis a partir dos dados armazenados são: séries históricas, súmula estatística, plano tabular, busca textual, estratificação dos grupos e anexos. A busca textual permite recuperar, dinamicamente, informações individualizadas sobre dados presentes na base do Diretório. De acordo com as séries históricas do CNPq, o Censo 2000 cobriu 224 instituições e 11.760 grupos de pesquisa; no Censo 2002, a cobertura do CN Pq passou para 268 instituições e 15.158 grupos; e no Censo de 2004, a cobertura foi de 335 instituições e19.470 grupos. Em número de instituições cobertas, 0 aumento do Censo de 2000 para 2002 foi de $20 \%$, enquanto 0 aumento do Censo de 2002 para 2004 foi de $25 \%$. 0 aumento de instituições cobertas pelo CN Pq entre o Censo de 2000 e o de 2004 foi de $50 \%$.

Para atingir os objetivos desejados, realizouseo levantamento e a análise da produção científica etecnológica sobre vigilância sanitária de 1997 a 2003 e a determinação do perfil dos grupos de pesquisa que trabalham com o tema nas bases de dados da Plataforma Lattes.

0 trabalho foi dividido em dois momentos por meio de consulta realizada via Internet. No primeiro momento, realizado de maio a julho de 2004, a busca ocorreu com os Censos de 2000 e 2002, em duas etapas. Na primeira, a busca da produção científica e, na segunda, a determinação dos grupos de pesquisa que trabal ham com vigilância sanitária. No segundo momento, realizado de abril a outubro de 2007, os dados da busca anterior foram atualizados com o Censo de2004.

$\mathrm{Na}$ primeira etapa da pesquisa censitária foi realizada busca textual, com o termo exato " $\mathrm{Vi}$ gilância Sanitária" e considerando-se os campos
"Nome do grupo", "Referência bibliográfica", "Título da produção C,T\&A" e "Palavra-chave da produção".

A produção científica foi classificada em categorias definidas pela própria base como:

Produção Bibliográfica: artigo completo publicado em periódicos especializados (circulação nacional); artigo completo publicado em periódicos especializados (circulação internacional); trabalhos completos publicados em anais deeventos científicos, tecnológicos eartísticos; livro; capítulo de livro; resumo de trabal hos publicados em revistas técnico-científicas; resumos de trabalhos publicados em anais de eventos científicos, tecnológicos e artísticos; outras publicações bibliográficas.

Orientações Concluídas: dissertação de mestrado; tese de doutorado; monografia de conclusão de curso de aperfeiçoamento/especialização; trabalho de conclusão de curso de graduação; iniciação científica.

Foram reclassificados os registros que estavam em categoria equivocada e foram excluídas as categorias de cunho não científico (Produção Técnica, Demais trabalhos e Produção artística e cultural).

Os diferentes Censos foram agrupados, sendo retiradas as duplicações existentes entre os Censos eno mesmo Censo entre suas categorias. Os dados foram analisados segundo 0 ano de publicação no período de 1997 a 2003 . 0 primeiro momento da busca de dados (2004) retornou 233 registros no Censo 2000 e 1.049 no Censo 2002; o segundo momento (2007) retornou 458 registros no Censo de2000, 831 no Censo de 2002 e 1.419 no Censo de 2004.

Na segunda etapa realizou-seuma "busca textual" do termo exato "Vigilância Sanitária", considerando-se os campos "Nome do grupo", "Nome da linha de pesquisa", "Palavra-chave da linha de pesquisa" e "Título da produção C,T\&A", "Palavra-chave da produção", "Repercussões do grupo". A primeira busca de dados (2004) retornou 152 registros no Censo 2000 e 339 no Censo 2002; a segunda busca (2007), 184 registros no Censo de 2000, 311 no Censo de 2002 e 538 no Censo de 2004. Foram retiradas as duplicações de registros no período.

Os dados obtidos foram analisados e validados, com a consulta em cada um dos currículos Lattes dos pesquisadores que registraram alguma produção em vigilância sanitária e em cada ficha dos grupos de pesquisa cuja produção tinha relação com a vigilância sanitária. 


\section{ResultadoseDiscussão}

A produção científica e tecnológica em vigilância sanitária

A Tabela 1 apresenta os registros válidos classificados nas devidas categorias de produção científica e ano de publicação.

Foram encontradas 1.194 produções no pe ríodo de estudo. Ao se considerar a produção anual total, verifica-se que houve um aumento de $540 \%$ de 1997 a 2003 (48/258 registros). H ouve aumento de cerca de $560 \%$ na produção bibliográfica ede $620 \%$ nas orientações concluídas (Gráfico 1).

Esses resultados confirmam a maior visibilidade da vigilância sanitária na saúde coletiva, como objeto de estudo3.

A preocupação com a formação dos trabaIhadores em vigilância sanitária tem tido expressão, ainda que tímida, desde o final dos anos $80 \mathrm{e}$ o início da década de 1990. N essa época, esforços foram feitos para a capacitação de trabalhadores

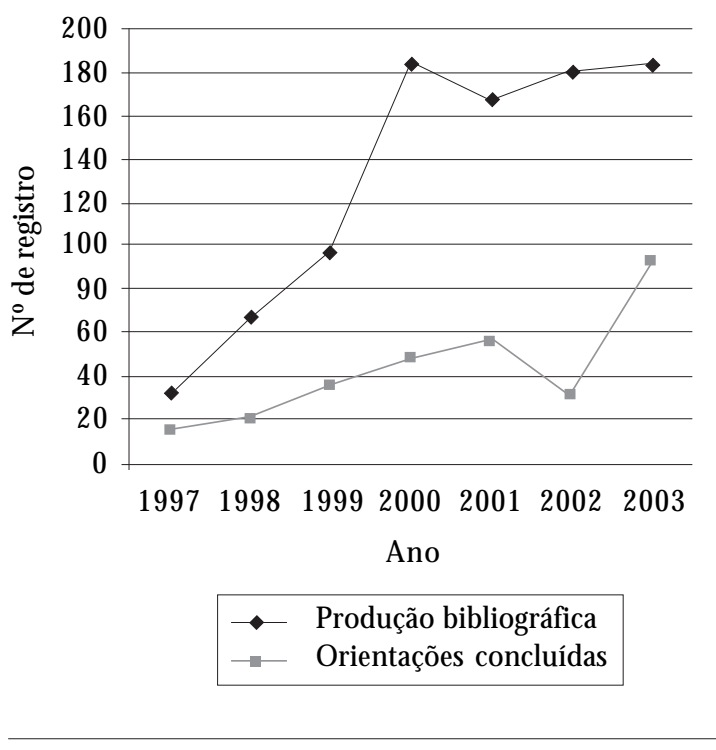

Gráfico 1. Distribuição $(\mathrm{n})$ da produção científica em vigilância sanitária de acordo com as categorias, por ano. Brasil, 1997-2003.

Tabela 1. Distribuição do tipo de produção científica em vigilância sanitária (n) de acordo com a categoria, por ano. Brasil, 1997-2003.

\begin{tabular}{|c|c|c|c|c|c|c|c|c|}
\hline \multirow[t]{2}{*}{ Tipo de produção } & \multicolumn{7}{|c|}{ Ano } & \multirow[t]{2}{*}{ Total } \\
\hline & 1997 & 1998 & 1999 & 2000 & 2001 & 2002 & 2003 & \\
\hline Total de produção bibliográfica & 33 & 67 & 97 & 184 & 168 & 180 & 184 & 913 \\
\hline $\begin{array}{l}\text { Artigo completo publicado em periódicos } \\
\text { especializados (circulação nacional) }\end{array}$ & 8 & 6 & 16 & 21 & 12 & 24 & 20 & 107 \\
\hline $\begin{array}{l}\text { Artigo completo publicado em periódicos } \\
\text { especializados (circulação internacional) }\end{array}$ & 0 & 3 & 5 & 14 & 9 & 7 & 13 & 51 \\
\hline $\begin{array}{l}\text { Trabalhos completos publicados em anais de } \\
\text { eventos científicos, tecnológicos e artísticos }\end{array}$ & 1 & 10 & 10 & 14 & 14 & 17 & 15 & 81 \\
\hline Livro & 0 & 1 & 1 & 2 & 0 & 0 & 0 & 4 \\
\hline Capítulo de livro & 1 & 2 & 2 & 12 & 5 & 7 & 8 & 37 \\
\hline $\begin{array}{l}\text { Resumo de trabalhos publicados em revistas } \\
\text { técnico-científicas }\end{array}$ & 2 & 0 & 7 & 6 & 11 & 15 & 19 & 60 \\
\hline $\begin{array}{l}\text { Resumos de trabalhos publicados em anais de } \\
\text { eventos científicos, tecnológicos e artísticos }\end{array}$ & 19 & 36 & 44 & 100 & 97 & 98 & 89 & 483 \\
\hline Outras publicações bibliográficas & 2 & 9 & 12 & 15 & 20 & 12 & 20 & 90 \\
\hline Total das orientações concluídas & 15 & 21 & 36 & 48 & 56 & 31 & 74 & 281 \\
\hline Dissertação de mestrado & 0 & 9 & 7 & 8 & 12 & 9 & 18 & 63 \\
\hline Tese de doutorado & 0 & 1 & 1 & 1 & 1 & 1 & 2 & 7 \\
\hline $\begin{array}{l}\text { Monografia de conclusão de curso de } \\
\text { aperfeiçoamento/especialização }\end{array}$ & 13 & 8 & 17 & 28 & 27 & 14 & 35 & 142 \\
\hline Trabalho de conclusão de curso de graduação & 0 & 1 & 3 & 7 & 9 & 6 & 11 & 37 \\
\hline Iniciação científica & 2 & 2 & 8 & 4 & 7 & 1 & 8 & 32 \\
\hline Total & 48 & 88 & 133 & 232 & 224 & 211 & 258 & 1.194 \\
\hline
\end{tabular}

Fonte: CN Pq - Diretório dos Grupos de Pesquisa no Brasil, Censos 2000, 2002 e 2004. 
por meio de cursos de pós-graduação lato sensu em vigilância sanitária como, por exemplo, os Cursos de Aperfeiçoamento em Vigilância Sanitária (Cavisa) e os Cursos de Especialização em Vigilância Sanitária, realizados simultaneamente na Escola Nacional de Saúde Pública da Fundação Oswaldo Cruz (Ensp/Fiocruz) e na Universidade de $M$ aringá (PR). H ouve, igualmente, a inclusão da temática nos Cursos de Especialização em Saúde Pública da Ensp/Fiocruz, realizados in locu ed eforma descentralizada. M ais recentemente, com a parceria da Agência Nacional de Vigilância Sanitária, foram criados Centros Colaboradores em Vigilância Sanitária (Cecovisa) em instituições acadêmicas, para fomentar também a realização de pesquisas de interesseda vigilância sanitária e o aumento da produção acadêmica no strictu senso. Essas experiências provavelmente se refletem na produção de resumos em congressos e de monografias, dissertações e teses.

A realização da I Conferência de Vigilância Sanitária, em 2001, reforçou, segundo Costa ${ }^{3}$ a vigilância sanitária como objeto de estudo e campo de prática ede produção de saberes. A instituição do Grupo Temático de Vigilância Sanitária (GTVisa) na Associação Brasileira de Pós-Graduação em Saúde Coletiva (Abrasco) passou a congregar docentes, pesquisadores, discenteseprofissionais, a fim de promover e incentivar trabaIhos científicos na área ${ }^{3}$, que puderam ter maior expressão com a realização dos Simpósios Brasileiros de Vigilância Sanitária (Simbravisa) e de outros eventos científicos específicos da área.

Estudo de caracterização da produção científica de líderes de grupos de pesquisa no campo da saúde, no Brasil, identificou que a apresentação de trabal hos em congressos nacionais seguida de apresentação em congresso internacional foram os tipos de produção mais frequentes. Em seguida, arrolam-se os artigos publicados em periódicos e livros ou capítulos de livros 8 . No presente estudo, os resumos de trabal hos publicados em anais deeventos científicos, tecnológicoseartísticos foram, dentre os itens da produção bibliográfica, os que tiveram o maior crescimento.

Cabe uma referência à limitação do estudo oriunda da utilização da Plataforma Lattes, sobretudo relativa à subnotificação das informações. Assim é que, por exemplo, os resumos publicados em evento específico da vigilância sanitária, o I Simpósio Brasileiro de Vigilância Sanitária, realizado em São Paulo em 2002, foram em número de 378 trabal hos, enquanto apenas cem foram registrados na Plataforma Lattes ${ }^{9}$. No banco de teses da Comissão de A perfei çoamento de Pessoal de Nível Superior (Capes), com o descritor vigilância sanitária, foram encontradas 29 teses e 133 dissertações no período de 1997 a 2003, enquanto na Plataforma Lattes foram registradas 7 e 63, respectivamente.

Rapini ${ }^{10}$ destaca a necessidade de atualização efornecimento de informações precisas para alimentar esse importante banco de dados. Outra questão relevante é a veracidade das informações fornecidas para esse banco de dados, tendo em vista que não há um mecanismo de validação dos dados informados.

Não é objeto deste trabalho a discussão da Saúde Pública/Coletiva ${ }^{11,12}$ ou o debate sobre a vigilância da saúde em suas três vertentes (de análise da situação de saúde, de integração institucional entre as vigilâncias ou de redefinição das práticas sanitárias) ${ }^{11}$ e o reflexo que a aplicação que esta proposição tem trazido às diferentes "vigilâncias". Entretanto, vale aqui referir que a mai or visibilidade da vigilância sanitária, nas últimas duas décadas, acompanha o esforço de (re) construção teórica do campo da SaúdeColetiva no Brasil. Ainda que al guns autores considerem a vigilância sanitária como um dos componentes da chamada "vigilância da saúde"11,13,14, este trabal ho considera quea vigilância sanitária possui suas especificidades e, com Dallari ${ }^{15}$, expressa um subcampo do conhecimento científico dotado deleis próprias, derivadas dos agentes einstituições que o caracterizam - que facilita a superação da divisão (hoje inconveniente) entre ciência pura e aplicada, capaz de aliar à práxis a produção e a reprodução do saber científico.

O perfil dos grupos de pesquisa com produção em vigilância sanitária

Número e formação dos pesquisadores

Foram encontrados 735 grupos de pesquisa com produção em vigilância sanitária, com um total de6.263 pesquisadores, sendo $4.095(65,4 \%)$ doutores, 1.577 mestres (25,2\%), $358(5,7 \%)$ especialistas, $194(3,1 \%)$ graduados e $40(0,6 \%)$ não informados. Identificaram-se 5.452 estudantes, sendo 1.055 (19,4\%) dedoutorado, 1.450 (26,6\%) demestrado, 171 (3,2\%) deespecialização, 2.016 (36,9\%) de graduação e 760 (13,9\%) não informados, além de 1.220 técnicos. Vale ressaltar que a proporção de pesquisadores doutores do número total de grupos de pesquisa do Diretório de Pesquisa da Plataforma Lattes foi de $61,8 \%$, num total de 77.649 pesquisadores existentes em 19.470 grupos de pesquisa nas séries históricas do CN Pq, no Censo de 2004. 
Os grupos de pesquisa

em vigilância sanitária

A formação de novos grupos que referem produzir em vigilância sanitária tem sido crescente. Foram criados 43 grupos (5,6\%) até 1982 , $39(5,3 \%)$ de 1983 a 1987, $84(11,4 \%)$ de 1988 a $1992,186(25,3 \%)$ de 1993 a $1997,210(28,9 \%)$ de 1998 a 2001 , e 173 (23,5\%) de 2002 a 2003. Prado eSayd ${ }^{16}$, em uma pesquisa utilizando igualmente o Diretório dos Grupos de Pesquisa do CN Pq, na linha de pesquisa sobre envelhecimento identificaram a criação de 144 grupos até 2000 . A opção deste trabalho foi a de identificar qualquer produção em vigilância sanitária enão ape nas as produções que possuíam linhas de pesquisa no tema específico, o que pode ter influenciado no alto número de grupos encontrados.

No formulário de coleta de dados do Dire tório dos Grupos de Pesquisa, o líder define a posição do grupo na árvore do conhecimento, indicando a grandeárea e a área de conhecimento predominante do grupo. Os grupos voltados para a pesquisa em vigilância sanitária são predominantemente $(49,9 \%)$ da área de Ciências da Saúde, seguida da de Ciências Agrárias (17,4\%) e das Ciências Biológicas (10,6\%).

A distribuição detalhada dos grupos de pesquisa em vigilância sanitária, por área de conhecimento, mostra que, apesar de a vigilância sanitária ser tema de grupos de diversas áreas, ela está concentrada em algumas delas. A área de Saúde Coletiva concentra $182(24,7 \%)$ dos grupos, seguido de Ciência eTecnologia deAlimentos com 57 (7,7\%), Farmácia com 55 (7,5\%), M edicina com 44 (6,0\%) e M edicina Veterinária com 39 (5,3\%). Esse resultado foi esperado, visto que abrange áreas de tradicional proximidade com as práticas de vigilância sanitária. Jáa epidemiologiatem forteinserção dos grupos (64\%) na área da Saúde Coletiva ${ }^{17}$.

$\mathrm{Na}$ Tabela 2 é possível visualizar os grupos segundo o seu ano de criação e as áreas de conhecimento.

Uma observação pertinente quanto aos grupos de pesquisa foi ressaltada por Prado e Sayd ${ }^{16}$. A análise dos grupos de pesquisa pelo seu ano de criação exige certa cautela, já que a pesquisa no assunto desejado podenão ter começado na mesma época em que o grupo foi criado. Pode ainda nem ter sido feita, pois o fato de o tema ser mencionado como linha de pesquisa pode representar apenas a intenção de realizar estudos futuros sobre 0 assunto.

Observa-se, para a vigilância sanitária, uma pluralidade de disciplinas a ela afeitas. Inserida no campo da Saúde Coletiva e voltada à prote- ção e promoção da saúde, agindo sobre múltiplos objetos, écompreensível a miríade de ciências dedicadas ao seu estudo. N esse sentido, sendo a vigilância sanitária um campo de prática, podese supor, com Campos ${ }^{12}$, certa sobreposição dos limites entre essas distintas disciplinas, conformando-se como um campo interdisciplinar e multiprofissional.

Regiões geográficas

e unidades da federação

É possível observar que a distribuição dos grupos que trabalham com vigilância sanitária por regiões geográficas e unidades da federação é desigual.

A Tabela 3 mostra a distribuição dos grupos depesquisa em vigilância sanitária, de 1997 a 2004, segundo as regiões geográficas e as unidades da federação. As regiões Sudeste e Sul concentram $76 \%$ dos grupos do país, sendo a maior concentração na região Sudeste $(53,2 \%)$ e nos estados deSão Paulo e Rio deJ aneiro (46,4\%). Resultado semelhante foi encontrado em pesquisa acerca da produção científica sobre o ambiente na SaúdeColetiva, que identificou serem as regiões Sudeste eSul as com maior número de instituições de pesquisa ${ }^{18}$.

A região N ordestereúne 17,3\% (127) dosgrupos que pesquisam em vigilância sanitária, seguida das regiões Centro- 0 este, com 5,2\% (38), e Norte, com 1,4\% (11). A distribuição pelas regiões segue o padrão do total de grupos de pesquisa presentes no Diretório, referente ao Censo de 2004. A região Sudeste foi igualmente a que mais concentrou grupos de pesquisa com enfoque em envelhecimento humano e epidemiologia ${ }^{10,19}$. Um dado que chama a atenção é que das 27 unidades federadas quatro não tinham grupo de pesquisa voltado diretamente para vigilância sanitária e seis tinham apenas um grupo com algum tipo de relação com o tema.

Instituições de pesquisa

A busca realizada no CNPq revelou que os 735 grupos de pesquisa com interesseem vigilância sanitária identificados pelo estudo estão distribuídos por 130 instituições de ensino e pesquisa do país. Dentre elas, é possível destacar a Universidade de São Paulo (USP) com 9,7\% (71) dos grupos, a Fundação Oswaldo Cruz (Fiocruz) com 9,1\% (67) dos grupos, a Universidade Federal de Santa Catarina (UFSC) com 3,9\% (29), a Universidade Estadual de Londrina (UEL), a Universidade Federal do Rio Grande do Sul (UFRGS) e a Universidade Estadual Paulista (Unesp), todas 
Tabela 2. Distribuição (n) dos grupos de pesquisa em vigilância sanitária, no Diretório dos Grupos de Pesquisa no Brasil, segundo as áreas de conhecimento por ano de criação do grupo. Brasil, 1997-2003.

\begin{tabular}{|c|c|c|c|c|c|c|c|}
\hline \multirow[t]{2}{*}{ Área de conhecimento } & \multicolumn{6}{|c|}{ Ano } & \multirow[t]{2}{*}{ Tota } \\
\hline & $\begin{array}{c}\text { Até } \\
1979\end{array}$ & $\begin{array}{l}1980- \\
1984\end{array}$ & $\begin{array}{l}1985- \\
1989\end{array}$ & $\begin{array}{l}1990- \\
1994\end{array}$ & $\begin{array}{l}1995- \\
1999\end{array}$ & $\begin{array}{l}2000- \\
2003\end{array}$ & \\
\hline Total de Ciências da Saúde & 7 & 12 & 28 & 63 & 107 & 150 & 367 \\
\hline Saúde Coletiva & 3 & 6 & 14 & 27 & 58 & 74 & 182 \\
\hline Farmácia & 0 & 2 & 1 & 10 & 18 & 24 & 55 \\
\hline M edicina & 3 & 3 & 2 & 8 & 6 & 22 & 44 \\
\hline Enfermagem & 0 & 1 & 4 & 8 & 9 & 14 & 36 \\
\hline Nutrição & 0 & 0 & 4 & 8 & 9 & 8 & 29 \\
\hline Outros & 1 & 0 & 3 & 2 & 7 & 8 & 21 \\
\hline Total de Ciências Agrárias & 3 & 7 & 9 & 23 & 34 & 52 & 128 \\
\hline Ciência e Tecnologia de Alimentos & 3 & 2 & 6 & 12 & 14 & 20 & 57 \\
\hline M edicina Veterinária & 0 & 3 & 2 & 8 & 9 & 17 & 39 \\
\hline Zootecnia & 0 & 0 & 0 & 1 & 6 & 10 & 17 \\
\hline Agronomia & 0 & 2 & 1 & 2 & 4 & 4 & 13 \\
\hline Engenharia Agrícola & 0 & 0 & 0 & 0 & 1 & 1 & 2 \\
\hline Total de Ciências Biológicas & 6 & 4 & 11 & 9 & 20 & 28 & 78 \\
\hline M icrobiologia & 0 & 0 & 2 & 5 & 5 & 10 & 22 \\
\hline Parasitologia & 0 & 0 & 3 & 0 & 1 & 6 & 10 \\
\hline Farmacologia & 0 & 1 & 3 & 2 & 2 & 2 & 10 \\
\hline Genética & 1 & 1 & 2 & 1 & 1 & 2 & 8 \\
\hline Imunologia & 1 & 0 & 0 & 0 & 2 & 3 & 6 \\
\hline Outros & 4 & 2 & 1 & 1 & 9 & 5 & 22 \\
\hline Total de Ciências Humanas & 0 & 1 & 2 & 8 & 15 & 31 & 57 \\
\hline Educação & 0 & 0 & 0 & 1 & 5 & 12 & 18 \\
\hline Sociologia & 0 & 1 & 2 & 3 & 6 & 7 & 19 \\
\hline Psicologia & 0 & 0 & 0 & 1 & 1 & 4 & 6 \\
\hline Filosofia & 0 & 0 & 0 & 1 & 0 & 4 & 5 \\
\hline Ciência Política & 0 & 0 & 0 & 0 & 2 & 2 & 4 \\
\hline Outros & 0 & 0 & 0 & 2 & 1 & 2 & 5 \\
\hline Total de Engenharias e Ciências da Computação & 2 & 2 & 3 & 10 & 14 & 12 & 43 \\
\hline Engenharia Sanitária & 1 & 1 & 0 & 2 & 5 & 5 & 14 \\
\hline Engenharia Biomédica & 0 & 1 & 0 & 0 & 5 & 0 & 6 \\
\hline Engenharia de Produção & 0 & 0 & 1 & 3 & 1 & 2 & 7 \\
\hline Engenharia Civil & 1 & 0 & 0 & 1 & 1 & 1 & 4 \\
\hline Engenharia Nuclear & 0 & 0 & 2 & 1 & 1 & 0 & 4 \\
\hline Outros & 0 & 0 & 0 & 3 & 1 & 4 & 8 \\
\hline Total de Ciências Sociais Aplicadas & 0 & 0 & 1 & 3 & 8 & 21 & 33 \\
\hline Direito & 0 & 0 & 1 & 0 & 3 & 9 & 13 \\
\hline Administração & 0 & 0 & 0 & 1 & 3 & 4 & 8 \\
\hline Serviço Social & 0 & 0 & 0 & 1 & 2 & 4 & 7 \\
\hline Comunicação & 0 & 0 & 0 & 1 & 0 & 2 & 3 \\
\hline Arquitetura e U rbanismo & 0 & 0 & 0 & 0 & 0 & 1 & 1 \\
\hline Demografia & 0 & 0 & 0 & 0 & 0 & 1 & 1 \\
\hline Total de Ciências Exatas e da Terra & 3 & 1 & 2 & 7 & 6 & 8 & 27 \\
\hline Química & 3 & 1 & 0 & 3 & 0 & 2 & 9 \\
\hline Ciência da Computação & 0 & 0 & 0 & 1 & 2 & 1 & 4 \\
\hline Geociências & 0 & 0 & 0 & 1 & 2 & 1 & 4 \\
\hline Probabilidade e Estatística & 0 & 0 & 0 & 1 & 0 & 2 & 3 \\
\hline Ciências da Informação & 0 & 0 & 0 & 0 & 2 & 1 & 3 \\
\hline Outros & 0 & 0 & 2 & 1 & 0 & 1 & 4 \\
\hline Total de Linguística, Letras e Artes & 0 & 0 & 0 & 1 & 0 & 1 & 2 \\
\hline Letras & 0 & 0 & 0 & 1 & 0 & 1 & 2 \\
\hline Total & 21 & 27 & 56 & 124 & 204 & 303 & 735 \\
\hline
\end{tabular}

Fonte: CN Pq - Diretório dos Grupos de Pesquisa no Brasil, Censos 2000, 2002 e 2004. 
Tabela 3. Distribuição dos grupos de pesquisa no Diretório dos Grupos de Pesquisa no Brasil e em vigilância sanitária, de acordo com as regiões geográficas, segundo as unidades da federação. Brasil, 1997-2004.

\begin{tabular}{|c|c|c|c|c|c|c|c|c|}
\hline \multirow{2}{*}{$\begin{array}{l}\text { Região geográfica/ } \\
\text { Unidade da federação }\end{array}$} & \multicolumn{4}{|c|}{ Grupos de pesquisa Diretório* } & \multicolumn{4}{|c|}{ Grupos de pesquisa em vigilância sanitária } \\
\hline & $\mathrm{n}$ & $\%$ & $\mathrm{n}$ & $\%$ & $\mathrm{n}$ & $\%$ & $\mathrm{n}$ & $\%$ \\
\hline Região Sudeste & 10.221 & 52,5 & & & 391 & 53,2 & & \\
\hline São Paulo & & & 5.541 & 28,5 & & & 188 & 25,6 \\
\hline Rio deJaneiro & & & 2.786 & 14,3 & & & 153 & 20,8 \\
\hline Minas Gerais & & & 1.694 & 8,7 & & & 49 & 6,7 \\
\hline Espírito Santo & & & 200 & 1 & & & 1 & 0,1 \\
\hline Região Sul & 4.580 & 23,5 & & & 168 & 23,2 & & \\
\hline Rio Grande do Sul & & & 2.072 & 10,6 & & & 68 & 9,3 \\
\hline Paraná & & & 1.512 & 7,8 & & & 54 & 7,3 \\
\hline Santa Catarina & & & 996 & 5,1 & & & 46 & 6,3 \\
\hline Região Centro-Oeste & 1.139 & 5,9 & & & 38 & 5,2 & & \\
\hline Distrito Federal & & & 477 & 2,4 & & & 19 & 2,6 \\
\hline Goiás & & & 266 & 1,4 & & & 13 & 1,8 \\
\hline Mato Grosso & & & 171 & 0,9 & & & 5 & 0,7 \\
\hline M ato Grosso do Sul & & & 225 & 1,2 & & & 1 & 0,1 \\
\hline Região N ordeste & 2.760 & 14,1 & & & 127 & 17,0 & & \\
\hline Bahia & & & 728 & 3,7 & & & 33 & 4,5 \\
\hline Pernambuco & & & 602 & 3,1 & & & 31 & 4,2 \\
\hline Ceará & & & 423 & 2,2 & & & 29 & 3,9 \\
\hline Paraíba & & & 329 & 1,7 & & & 13 & 1,8 \\
\hline Rio Grande do Norte & & & 220 & 1,1 & & & 11 & 1,5 \\
\hline M aranhão & & & 119 & 0,6 & & & 8 & 1,1 \\
\hline Alagoas & & & 133 & 0,7 & & & 1 & 0,1 \\
\hline Piauí & & & 101 & 0,5 & & & 1 & 0,1 \\
\hline Sergipe & & & 105 & 0,5 & & & 0 & 0 \\
\hline Região N orte & 770 & 4 & & & 11 & 1,4 & & \\
\hline Pará & & & 286 & 1,5 & & & 5 & 0,7 \\
\hline Amazonas & & & 289 & 1,5 & & & 4 & 0,5 \\
\hline Roraima & & & 30 & 0,1 & & & 1 & 0,1 \\
\hline Rondônia & & & 33 & 0,2 & & & 1 & 0,1 \\
\hline Tocantins & & & 97 & 0,5 & & & 0 & 0 \\
\hline Acre & & & 25 & 0,1 & & & 0 & 0 \\
\hline Amapá & & & 10 & 0,05 & & & 0 & 0 \\
\hline Total geral & 19.470 & 100 & 19.470 & 100 & 735 & 100 & 735 & 100 \\
\hline
\end{tabular}

Fonte: CN Pq - Diretório dos Grupos de Pesquisa no Brasil, Censos 2000, 2002 e 2004.

*Foi considerado o número total degrupos de pesquisa presenteno Diretório referente ao Censo de 2004.

com 3,2\% (24). A Universidade Federal do Rio de Janeiro (UFRJ) apareceem sétimo lugar, com 3,1\% (23) dos grupos. A USP também aparece como a instituição que mais concentra grupos com enfoque em envelhecimento e ocupa o segundo lugar quando o enfoqueéepidemiologia. A Fiocruz éa quemais concentra grupos em epidemiologia ${ }^{10,11}$.

Os grupos de pesquisa do CN Pq concentram suas atividades em universidades, em instituições de ensino superior e em institutos que ministram cursos de pós-graduação. Outro fator que merece destaqueéo numero deinstituições públicas, tanto de ensino como de pesquisa, encontrado no estudo, confirmando assim resultados obtidos em outros estudos no campo da Saúde Coletiva ${ }^{18,20}$.

No quese refereà vigilância sanitária, tal fato pode ser reflexo do investimento e da demanda dos serviços de vigilância sanitária buscando conhecimento e aperfeiçoamento da área e, assim, capacitação de seus profissionais; além disso, por tratar-se de uma atividade da saúde eminentemente pública e estatal. 


\section{Considerações finais}

Houve, no período estudado, um aumento na produção científica em vigilância sanitária e no número de grupos de pesquisa que estudam o tema. Pode-se perceber, igualmente, a diversidade de disciplinas envolvidas nessa produção, permitindo caracterizar a vigilância sanitária como um campo de prática ede produção de conhecimento interdisciplinar emultiprofissional. Entretanto, os grupos concentram-se em al gumas regiões e especificamenteem alguns estadoseem certas áreas de conhecimento. Além dos fatores gerais que condicionam o crescimento da totalidade de pesquisa em saúde no Brasil, é possível associar esses dados à ideia de que a vigilância sanitária vem se constituindo, efetivamente, como uma área de produção de conhecimento. Pode-se supor que fatores tais como o reconhecimento da vigilância sanitária no campo da Saúde Coletiva eo investimento sistemático e regular nas ações, além do incentivo na produção de conhecimento da área, após a criação da Agência N acional de Vigilância Sanitária, tenham contribuído para 0 aumento verificado. Ainda assim, a produção científica encontrada na fonte de dados utilizada, a Plataforma Lattes, parece ser insuficiente, dada a importância do conhecimento científico ante a sua natureza de ação e a amplitude das atribuições da vigilância sanitária.

Há, entretanto, que se registrar a possível limitação da população informantee da possibilidade desub-registro nasinformações, principal- mente da produção realizada nos serviços de saúde, visto ser a vigilância sanitária importante campo de prática, portanto, de produção de conhecimento fora das instituições acadêmicas. 0 preenchimento das informações nos currículos, realizado a partir do autorreconhecimento dos pesquisadores e estudantes, pode também influenciar no baixo registro, uma vez que apenas mais recentemente a vigilância sanitária tem ganhado mai or visibilidade.

Algumas limitações do estudo dizem respeito à fonte de dados utilizada, sobretudo na classificação da produção registrada, sendo uma parte delas superada pela consulta a cada um dos currículos dos pesquisadores. Ainda assim, évaliosa fonte e única disponível on-line capaz de proporcionar os dados desejados. Um mecanismo de validação dos dados no momento do cadastro do currículo ou da ficha do grupo de pesquisa poderia aumentar a qualidade e a confiabilidade dos dados disponíveis no Diretório de Pesquisa do CNPq.

Finalmente, a vigilância sanitária, definida como conjunto de ações capaz de eliminar, diminuir ou prevenir riscos à saúde e de intervir nos problemas sanitários decorrentes do meio ambiente, da produção e circulação de bens e da prestação de serviços de interesse da saúde (art. $6^{\circ}$ parágrafo $\left.1^{0}\right)^{21}$, não deve apenas acompanhar o desenvolvimento científico-tecnológico, masnecessita criar um conhecimento que articule a ciência pura e aplicada ${ }^{15}$, para intervir no campo produtivo de forma a proteger a saúde da população.

\section{Colaboradores}

VLE Pepe trabalhou na concepção e no delineamento da análise, na redação do artigo e na aprovação da versão a ser publicada; ABM Noronha, na concepção e no delineamento, análise einterpretação dos dados e redação do artigo; TA Figueiredo, na análise e interpretação dos dados e redação do artigo; AAL Souza eDM Pontes Júnior, na análise e interpretação dos dados e redação inicial do artigo; CVS Oliveira, na redação do artigo.
Agradecimentos

Os autores agradecem o financiamento parcial do Centro de Vigilância Sanitária da Secretaria de Estado de Saúde do Rio de Janeiro, por meio do Projeto Apoio à Consolidação do Sistema Estadual deVigilância Sanitária, eà Agência N acional de Vigilância Sanitária, através do convênio com a Escola N acional de Saúde Pública Sergio Arouca, por meio do Centro Colaborador de Vigilância Sanitária (Cecovisa/Ensp), Pesquisa e Desenvolvimento Institucional em Vigilância Sanitária. 


\section{Referências}

1. Lucchese G. A vigilância sanitária no Sistema Único de Saúde. In: I Conferência Nacional de Vigilância Sanitária. Caderno de Textos. Brasília; 2001. p. 53-69.

2. Costa EA, Rozenfeld S. Constituição da vigilância sanitária no Brasil. In: Rozenfeld S, organizador. Fundamentos da vigilância sanitária. Rio de Janeiro: Editora Fiocruz; 2000. p. 15-40.

3. Costa EA, Fernandes TM, Pimenta TS. A vigilância sanitária nas políticas de saúde no Brasil e a construção da identidade de seus trabalhadores (19761999). Cien Saude Colet 2008; 13(3):995-1004.

4. Fórum Reflexão Universitária. Desafios da pesquisa do Brasil: uma contribuição ao debate. São Paulo em Perspectiva 2002; 16(4):15-23.

5. Paula M V. Explorando o potencial da Plataforma Lattes como fonte de conhecimento organizacional em Ciência e Tecnologia [dissertação]. Brasília: Universidade Católica de Brasília; 2004.

6. Agência Nacional de Vigilância Sanitária. Censo Nacional de Trabalhadores da Vigilância Sanitária, 2004. [acessado 2008 jul 22]. Disponível em: http:/ /www.anvisa.gov.br/hotsite/censo_2004/historico/ publicacao.pdf

7. Brasil. Ministério da Saúde. Plano Diretor de Vigilância Sanitária. [site da Internet] [acessado 2008 jul 21]. Disponível em: http://www.sgc.goias.gov.br/ upload/links/arq_907_PDVISA-181206.pdf

8. Silva TR, Szklo F, Barata RB, Noronha J. Avaliação do Sistema de Pesquisa em Saúde no Brasil: algumas características dos pesquisadores e produção científica. Rev Eletrônica de Comunicação, Informação e Inovação em Saúde 2007; 1(1):9-18.

9. Associação Brasileira de Pós-Graduação em Saúde Coletiva (Abrasco). I Simbravisa: Simpósio Brasileiro de Vigilância Sanitária - livro de resumos. Rev Bras Epidemiol 2002; Supl.Esp.:1-152.

10. Rapini MS. 0 diretório dos grupos de pesquisa do $\mathrm{CNPq}$ e a interação universidade-empresa no Brasil: uma proposta metodológica de investigação. $R$ Econ Contemp 2007; 11(1):99-117.

11. Teixeira CF, Paim JS, Vilasboas AL. SUS, modelos assistenciais e vigilância da saúde. Informe Epidemiológico do SUS 1998; 7(2):7-28.
12. Campos GWS. Saúde Pública e Saúde Coletiva: campo e núcleo de saberes e práticas. Cien Saude Colet 2000; 5(2):219-230.

13. M endes EV. A construção social da vigilância à saúde no Distrito Sanitário. Brasília: O pas; 1993. p. 7-19. (Série Desenvolvimento de Serviços de Saúde, 10).

14. Albuquerque MIN, Carvalho EM F, Lima LP. Vigilância rpidemiológica: conceitos e institucionalização. Revista Brasileira de Saúde M aterno-Infantil 2002; 2(1):7-14.

15. Dallari SG. A vigilância sanitária e a Saúde Coletiva. Rev Bras Epidemiol 2002; Supl.Esp.:11-15.

16. Prado SD, Sayd JD. A pesquisa sobre envelhecimento humano no Brasil: grupos e linhas de pesquisa. Cien Saude Colet 2004; 9(1):57-68.

17. Guimarães $R$, Lourenço $R$, Cosac $S$. A pesquisa em epidemiologia no Brasil. Rev Saude Publica 2001; 35(4):321-340.

18. Freitas CM . A produção científica sobre 0 ambiente na Saúde Coletiva. Cad Saude Publica 2005; 21(3):679-701.

19. Aquino EM. Epidemiologia e Saúde Coletiva no Brasil: desafios para a formação em pesquisa. Rev Bras Epidemiol 2008; 11(Supl.1):151-158.

20. Barreto ML. Crescimento e tendência da produção científica em epidemiologia no Brasil. Rev Saude Publica 2006; 40(Nº Esp.):79-85.

21. Brasil. Lei $\mathrm{n}-8.080$, de 19 de setembro de 1990. Dispõe sobre as condições para a promoção, proteção e recuperação da saúde, a organização e 0 funcionamento dos serviços correspondentes e dá outras providências. Diário Oficial da União 1991; 19 set.

Artigo apresentado em 09/02/2009

Aprovado em 28/08/2009

Versão final apresentada em 29/09/2009 